hep-th/0004093

\title{
Supersymmetrizing Branes with Bulk in Five-Dimensional Supergravity
}

\author{
Adam Falkowski ${ }^{1}$ Zygmunt Lalak $^{1,2}$ and Stefan Pokorski ${ }^{1}$ \\ ${ }^{1}$ Institute of Theoretical Physics \\ University of Warsaw, Poland \\ ${ }^{2}$ Physikalisches Institut, Universität Bonn \\ Nussallee 12, D-53115 Bonn, Germany
}

\begin{abstract}
We supersymmetrize a class of moduli dependent potentials living on branes with the help of additional bulk terms in $5 \mathrm{~d} N=2$ supergravity. The space of Poincare invariant vacuum solutions includes the Randall-Sundrum solution and the $M$-theoretical solution. After adding gauge sectors to the branes we discuss breakdown of low energy supersymmetry in this setup and hierarchy of physical scales. In the limit of large warp factors we find decoupling between effects stemming from different branes in the compactified theory.
\end{abstract}


The idea of higher-dimensional unification of the fundamental interactions attracts considerable interest and receives more and more concrete realizations. The general setup for such unification consists of hypersurfaces hosting various gauge sectors which are embedded into higher dimensional bulk space. Bulk interactions are those of higher dimensional gravity coupled to certain scalar and form fields, as well as to fermions, which are however inert with respect to gauge groups localized on branes. From the low-energy point of view most of the nontrivial features of field theoretical models that are related to spatial separation of gauge sectors should be clearly visible at the level of the simple five-dimensional theory. So far, the

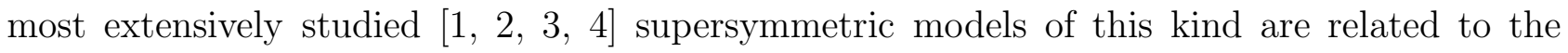
supergravity model constructed by Horava and Witten as the low energy effective theory of the strongly coupled heterotic $E_{8} \times E_{8}$ superstring in [5, 6] (see also [7]). In particular, a five-dimensional theory is the simplest nontrivial setup to study spontaneous supersymmetry breakdown and its transmission between the branes [3, [4, 8]. The agents of that transmission are the bulk fields.

Much attention to five-dimensional gravity has also been drawn by the recent observation that 5d anti-de-Sitter gravity with 3-branes embbedded in the bulk allows for solution with localized gravitational field [9]. However, in this scenario it is necessary to add the cosmological terms localized on the boundaries with coefficients determined uniquely by the cosmological term in the bulk. The correlation between bulk and boundary potentials is crucial for obtaining a consistent solution to Einstein equations, as well as vanishing of the cosmological constant in the effective four dimensional theory. In the original paper [10] no symmetry justifies this apparent fine-tuning.

A considerable effort has been devoted to supersymmetrization of the Randall-Sundrum model, mostly with negative results [11], [12]. The exception is the recent reference [13]. In that paper the authors start with $5 \mathrm{~d} \mathrm{~N}=2$ pure supergravity with a cosmological constant $\Lambda$ and demonstrate that inclusion of branes in a supersymmetric way leads to the Randall-Sundrum action. Neither scalar fields in the bulk nor gauge and matter on the branes are included in that construction.

The purpose of this paper is to study, in a more general way, a class of five dimensional locally supersymmetric theories with 3-branes. We demonstrate that certain types of potentials introduced on branes, that are not parts of a supersymmetric model on a brane, can be supersymmetrized by modifications of the $5 \mathrm{~d}$ supergravity in the bulk. The requirement of supersymmetry yields relations between bulk and brane cosmological potentials, such that in suitable limits we obtain a supersymmetric version of the Randall-Sundrum scenario or the M-theoretical solution. The general case can still preserve the characteristic features of the Randall-Sundrum model and at the same time includes non-trivial potentials for the hypermultiplets of the bulk theory. We find a vacuum solution which preserves one half of the supercharges. This solution can serve as a background for the compactification to the effective 4d theory with $\mathrm{N}=1$ supersymmetry. Next, we include gauge fields on the brane, and discuss the modification of the brane action and supersymmetry transformation laws, necessary to obtain a supersymmetric theory.

Finally, we discuss supersymmetry breaking and the role played by the warp factor and conclude the paper with some remarks on the consistency of the compactification to $4 \mathrm{~d}$. 
We start with a five-dimensional $\mathrm{N}=2$ supergravity on the manifold $M_{4} \times S_{1} / \mathbf{Z}_{2}$ which includes a gravity multiplet $\left(e_{\alpha}^{m}, \psi_{\alpha}, \mathcal{A}_{\alpha}\right)$ coupled to one hypermultiplet $\left(\lambda^{a}, V, \sigma, \xi, \bar{\xi}\right)$ forming a $S U(2,1) / U(2)$ non-linear sigma model. Two parallel 3 -branes are located at $x^{5}=0$ and $x^{5}=\pi \rho$. This particular framework is motivated by the Horava-Witten model compactified to 5d [1, []. The sigma-model metric can be read from the Kähler potential: $K=-\ln (S+\bar{S}-$ $2 \xi \bar{\xi}), S=V+\xi \bar{\xi}+i \sigma$. The conventions and normalizations we use are mainly those of reference [2]. The signature of the metric tensor is $(-++++)$. The $\mathrm{SU}(2)$ spinor indices are raised with antisymmetric tensor $\epsilon^{A B}$, and the $\operatorname{Sp}(1)$ indices (those of hyperino) with $\Omega^{a b}$. We choose $\epsilon^{12}=\epsilon_{12}=\Omega^{21}=\Omega_{21}=1$. The rule for dealing with symplectic spinors is ${\overline{\psi_{1}}}^{A} \psi_{2}^{B}={\overline{\psi_{2}}}^{B} \psi_{1}^{A}$ (note that $\bar{\psi}^{A}=\overline{\psi_{A}}$ ). The $\mathbf{Z}_{2}$ symmetry acts as reflection $x^{5} \rightarrow-x^{5}$ and is represented in such a way that bosonic fields $\left(e_{\mu}^{m}, e_{5}^{5}, \mathcal{A}_{5}, V, \sigma\right)$ are even, and $\left(e_{5}^{m}, e_{\mu}^{5}, \mathcal{A}_{\mu}, \xi\right)$ are odd. The indice $\alpha, \beta \ldots$ are five dimensional $(0 . .3,5)$, while $4 \mathrm{~d}$ indices are denoted by $\mu, \nu, \ldots$. The action of the $\mathbf{Z}_{\mathbf{2}}$ on fermion fields and on parameter $\epsilon$ of supersymmetry transformations is defined as:

$$
\begin{aligned}
& \gamma_{5} \psi_{\mu}^{A}\left(x^{5}\right)=\left(\sigma^{3}\right)^{A}{ }_{B} \psi_{\mu}^{B}\left(-x^{5}\right) \quad \gamma_{5} \psi_{5}^{A}\left(x^{5}\right)=-\left(\sigma^{3}\right)^{A}{ }_{B} \psi_{5}^{B}\left(-x^{5}\right) \\
& \gamma_{5} \lambda^{a}\left(x^{5}\right)=-\left(\sigma^{3}\right)^{a}{ }_{b} \lambda^{b}\left(-x^{5}\right) \quad \gamma_{5} \epsilon^{A}\left(x^{5}\right)=\left(\sigma^{3}\right)^{A}{ }_{B} \epsilon^{B}\left(-x^{5}\right)
\end{aligned}
$$

where $\gamma_{5}=\left(\begin{array}{rr}-1 & 0 \\ 0 & 1\end{array}\right), \sigma^{3}=\left(\begin{array}{cc}1 & 0 \\ 0 & -1\end{array}\right), A, a=1,2$. Symplectic Majorana spinors in $5 \mathrm{~d}$ satisfy $\bar{\chi}^{A}=$ $\left(C \chi^{A}\right)^{T}$ with $C=-i \gamma^{2} \gamma^{0}$ in $4 \mathrm{~d}$ chiral representation. The kinetic part of the action and supersymmetry transformation laws up to 3 -fermi terms are:

$$
\begin{gathered}
S=-\int d^{5} x e_{5} \frac{1}{2 \kappa^{2}}\left(R+\frac{3}{2} \mathcal{F}_{\alpha \beta} \mathcal{F}^{\alpha \beta}+\frac{1}{\sqrt{2}} \epsilon^{\alpha \beta \gamma \delta \epsilon} \mathcal{A}_{\alpha} \mathcal{F}_{\beta \gamma} \mathcal{F}_{\delta \epsilon}+\frac{1}{2 V^{2}}\left(\partial_{\alpha} V \partial^{\alpha} V+D_{\alpha} \sigma D^{\alpha} \sigma\right)\right. \\
+\frac{2}{V} \partial_{\alpha} \xi \partial^{\alpha} \bar{\xi}+\frac{i}{2 V^{2}}\left(\xi \partial_{\alpha} \bar{\xi} D^{\alpha} \sigma-\bar{\xi} \partial_{\alpha} \xi D^{\alpha} \sigma\right)-\frac{1}{2 V^{2}}\left(\left(\xi \partial_{\alpha} \bar{\xi}\right)^{2}+\left(\bar{\xi} \partial_{\alpha} \xi\right)^{2}-\left|\bar{\xi} \partial_{\alpha} \xi\right|^{2}\right) \\
\left.\left.-\left(\frac{1}{2} \frac{\psi_{\mu}^{1}}{\mu} \gamma^{\mu \nu \rho} D_{\nu} \psi_{\rho}^{1}+(1 \rightarrow 2)\right)-\left(\frac{1}{2} \overline{\lambda^{1}} \gamma^{\mu} D_{\mu} \lambda^{1}\right)+(1 \rightarrow 2)\right)\right) \\
\delta e_{\alpha}^{m}=\frac{1}{2} \overline{\epsilon^{1}} \gamma^{m} \psi_{\alpha}^{1}+(1 \rightarrow 2) \\
\delta \psi_{\alpha}^{1}=D_{\alpha} \epsilon^{1}-\frac{i}{4 \sqrt{2}}\left(\gamma_{\alpha}^{\beta \gamma}-4 \delta_{\alpha}^{\beta} \gamma^{\gamma}\right) \mathcal{F}_{\beta \gamma} \epsilon^{1}+\frac{i}{4 V} D_{\alpha} \sigma \epsilon^{1}+\frac{1}{4 V}\left(\xi \partial_{\alpha} \bar{\xi}-\bar{\xi} \partial_{\alpha} \xi\right) \epsilon^{1}-\frac{1}{\sqrt{V}} \partial_{\alpha} \xi \epsilon^{2} \\
\delta \psi_{\alpha}^{2}=D_{\alpha} \epsilon^{2}-\frac{i}{4 \sqrt{2}}\left(\gamma_{\alpha}^{\beta \gamma}-4 \delta_{\alpha}^{\beta} \gamma^{\gamma}\right) \mathcal{F}_{\beta \gamma} \epsilon^{2}-\frac{i}{4 V} D_{\alpha} \sigma \epsilon^{2}-\frac{1}{4 V}\left(\xi \partial_{\alpha} \bar{\xi}-\bar{\xi} \partial_{\alpha} \xi\right) \epsilon^{2}+\frac{1}{\sqrt{V}} \partial_{\alpha} \bar{\xi} \epsilon^{1} \\
\delta \mathcal{A}_{\alpha}=-\frac{i}{2 \sqrt{2}} \overline{\psi_{\alpha}^{1}} \epsilon^{1}+(1 \rightarrow 2) \\
\delta V=\frac{i}{\sqrt{2}} V\left(\overline{\epsilon^{1}} \lambda^{1}\right)-(1 \rightarrow 2) \\
\delta \sigma=+\frac{1}{\sqrt{2}} V\left(\overline{\epsilon^{1}} \lambda^{1}\right)+(1 \rightarrow 2)+\sqrt{\frac{V}{2}}\left(\xi \overline{\epsilon^{1}} \lambda^{2}-\bar{\xi} \overline{\epsilon^{2}} \lambda^{1}\right) \\
\delta \xi=-\frac{i \sqrt{V}}{\sqrt{2}}\left(\overline{\epsilon^{2}} \lambda^{1}\right) \quad \delta \bar{\xi}=-\frac{i \sqrt{V}}{\sqrt{2}}\left(\overline{\epsilon^{1}} \lambda^{2}\right) \\
\delta \lambda^{1}=-\frac{i}{2 \sqrt{2} V}(\not \partial(V+i \sigma)-\bar{\xi} \not \partial \xi+\xi \not \partial \bar{\xi}) \epsilon^{1}+\frac{i}{\sqrt{2 V}} \not \partial \xi \epsilon^{2} \\
\delta \lambda^{2}=+\frac{i}{2 \sqrt{2} V}(\not \partial(V-i \sigma)+\bar{\xi} \not \partial \xi-\xi \not \partial \bar{\xi}) \epsilon^{2}+\frac{i}{\sqrt{2 V}} \not \partial \bar{\xi} \epsilon^{1} .
\end{gathered}
$$

We assume a scalar potential $\delta\left(x^{5}\right) \frac{e}{\kappa^{2}}\left(-\Lambda+\frac{\sqrt{2} \alpha}{V}\right)$ localized on, say, the first brane (note the delta function), and study the variation of the brane action under supersymmetry transformations. The motivation for the constant $(\Lambda)$ part of this expression is that it will finally lead us to the 
Randall-Sundrum expotential solutions. At the same time we allow for cosmological potentials for hypermultiplet scalars; the above form is motivated by the M-theory example and is a natural extension in the presence of hypermultiplets. The generalizations are possible, but $\sigma$-dependent terms in the potential break the translational $\mathrm{U}(1)$ symmetry $\sigma \rightarrow \sigma+$ const which is useful when we introduce potential in the bulk, while $\xi$ cannot appear in the boundary potential because of parity assignments. We will be able to supersymmetrize this action by modification of the bulk action only (thus, our construction is alternative to [13]).

For simplicity, we initially put $\alpha=0$ and consider a cosmological term of the form:

$$
\mathcal{L}_{B}=-\delta\left(x^{5}\right) \frac{e}{\kappa^{2}} \Lambda
$$

where $e$ is 4 d determinant built from the metric induced on the brane. We wish to supersymmetrize this term. The supersymmetry variation of $\mathcal{L}_{B}$ comes from varying $e$ :

$$
\delta \mathcal{L}=+\frac{1}{2} \delta\left(x^{5}\right) e \Lambda\left(\overline{\psi_{\mu}^{1}} \gamma^{\mu} \epsilon^{1}+(1 \rightarrow 2)\right) .
$$

We observe that, without further modification of the boundary action, we can cancel this variation by modifying gravitino transformation law:

$$
\begin{gathered}
\delta \psi_{\alpha}^{1}=+\frac{\Lambda}{12} \epsilon\left(x^{5}\right) \gamma_{\alpha} \epsilon^{1} \\
\delta \psi_{\alpha}^{2}=-\frac{\Lambda}{12} \epsilon\left(x^{5}\right) \gamma_{\alpha} \epsilon^{2} .
\end{gathered}
$$

Note that these corrections are compatible with $\mathbf{Z}_{2}$ symmetry defined by (1).

If we vary $\psi$ in the gravitino kinetic term, the fifth derivative acting on the step function produces an expression multiplied by a delta function, which precisely cancels (6). But now the bulk theory is not supersymmetric. It is straightforward to show that the variations of the gravitino kinetic term resulting from (7) and proportional to $\Lambda \epsilon\left(x^{5}\right)$ can be cancelled by addding a 'gravitino mass term':

$$
\mathcal{L}_{\psi^{2}}=+\frac{e_{5}}{8 \kappa^{2}} \Lambda \epsilon\left(x^{5}\right)\left(\overline{\psi_{\alpha}^{1}} \gamma^{\alpha \beta} \psi_{\beta}^{1}-\overline{\psi_{\alpha}^{2}} \gamma^{\alpha \beta} \psi_{\beta}^{2} .\right)
$$

The gravitino variation $\delta \psi_{\alpha}^{A}=D_{\alpha} \epsilon^{A}$ in (8) cancels the above mentioned variation, but now (7) applied to the mass term (8) will produce a variation proportional to $\Lambda^{2}$, which can be cancelled by varying the determinat in a new 'cosmological term':

$$
\mathcal{L}_{C}=\frac{e_{5}}{6 \kappa^{2}} \Lambda^{2} .
$$

Moreover, in our framework, $\epsilon\left(x^{5}\right)$ has another discontinuity at $x^{5}=\pi \rho$ so an additional term multiplied by $\delta\left(x^{5}-\pi \rho\right)$ appears in the varied bulk Lagrangian. This variation can be cancelled by adding a cosmological term confined to that brane:

$$
\mathcal{L}_{B^{\prime}}=\delta\left(x^{5}-\pi \rho\right) \frac{e}{\kappa^{2}} \Lambda
$$


(The minus sign relative to (5) appears because $\epsilon\left(x^{5}\right)$ has a 'step down' at $x^{5}=\pi \rho$ ) 円. Note that the cosmological term appeared with a plus sign. The relevant part of the bulk action now reads $S=-\frac{1}{2} \int\left(R-\frac{1}{3} \Lambda^{2}\right)$ which allows for anti-de-Sitter solutions. In fact, the coefficient of (9) is precisely the one we need to obtain the Randall-Sundrum scenario, as we will show soon.

The above mentioned corrections are still not sufficient to supersymmetrize the bulk lagrangian. We also need the hyperino mass term:

$$
\mathcal{L}_{\lambda^{2}}=+\frac{e_{5}}{8 \kappa^{2}} \epsilon\left(x^{5}\right) \Lambda\left(\overline{\lambda^{1}} \lambda^{1}-(1 \rightarrow 2)\right)
$$

and the coupling of the graviphoton to gravitino:

$$
\mathcal{L}_{A}=-\frac{i e_{5}}{4 \sqrt{2} \kappa^{2}} \epsilon\left(x^{5}\right) \Lambda\left(\left(\overline{\psi^{1}}{ }_{\alpha} \gamma^{\alpha \beta \gamma} \psi_{\gamma}^{1}\right) \mathcal{A}_{\beta}-(1 \rightarrow 2)\right)
$$

In addition a graviphoton dependent correction to gravitino transformation law appears:

$$
\delta \psi_{\alpha}^{A}=+\frac{i}{2 \sqrt{2}} \epsilon\left(x^{5}\right) \Lambda\left(\sigma^{3}\right)_{B}^{A} \epsilon^{B} \mathcal{A}_{\alpha} .
$$

Further, we need 4-fermi terms in the bulk action to complete the supersymmetrization, but these are not given in this letter.

Let us now assume $\Lambda=0$ and consider the boundary term:

$$
\mathcal{L}=\delta\left(x^{5}\right) \frac{e}{\kappa^{2}} \frac{\sqrt{2} \alpha}{V}
$$

The variation of the determinant can be canceled by modifying $\delta \psi$, similarly to the previous case:

$$
\begin{gathered}
\delta \psi_{\alpha}^{1}=-\frac{\sqrt{2}}{12} \frac{\alpha}{V} \epsilon\left(x^{5}\right) \gamma_{\alpha} \epsilon^{1} \\
\delta \psi_{\alpha}^{2}=+\frac{\sqrt{2}}{12} \frac{\alpha}{V} \epsilon\left(x^{5}\right) \gamma_{\alpha} \epsilon^{2} .
\end{gathered}
$$

We must also vary the hyperplet modulus $V$ in (14) $\left(\delta V=\frac{i V}{\sqrt{2}}\left(\overline{\epsilon^{1}} \lambda^{1}-\overline{\epsilon^{2}} \lambda^{2}\right)\right)$

$$
\delta \mathcal{L}=-i \delta\left(x^{5}\right) e \frac{\alpha}{V}\left(\overline{\epsilon^{1}} \lambda^{1}-(1 \rightarrow 2)\right) .
$$

This variation can be cancelled by modifying supersymmetry transformation law of the hyperino $\lambda$ :

$$
\begin{gathered}
\delta \lambda^{1}=\frac{i}{2 V} \alpha \epsilon\left(x^{5}\right) \epsilon^{1} \\
\delta \lambda^{2}=\frac{i}{2 V} \alpha \epsilon\left(x^{5}\right) \epsilon^{2} .
\end{gathered}
$$

\footnotetext{
${ }^{1}$ The need for the bulk gravitino mass term proportional to $\epsilon\left(x^{5}\right)$ has been also pointed out in [14].
} 
A similar mechanism works: in the variation of the hyperino kinetic term the fifth derivative acts on the step function which leads to a term which precisely cancels (16). Note that it is only the potential $\alpha / V$ which causes the corrections to the hyperino transformation law. As before, we need to supersymmetrize further. Two-fermi terms and, as a consequence, a cosmological potential is necessary:

$$
\begin{gathered}
\mathcal{L}=i \frac{e_{5}}{2 V \kappa^{2}} \alpha \epsilon\left(x^{5}\right)\left(-\frac{\sqrt{2}}{4}\left(\overline{\psi_{\alpha}^{1}} \gamma^{\alpha \beta} \psi_{\beta}^{1}-(1 \rightarrow 2)\right)+\left(\overline{\lambda^{1}} \gamma^{\alpha} \psi_{\alpha}^{1}+(1 \rightarrow 2)\right)+\frac{3 \sqrt{2}}{4}\left(\overline{\lambda^{1}} \lambda^{1}-(1 \rightarrow 2)\right)\right) \\
\mathcal{L}_{C}=-\frac{e_{5}}{6 \kappa^{2}} \frac{\alpha^{2}}{V^{2}} .
\end{gathered}
$$

However, this time a minus sign relative to that of (9) appears, and anti-de-Sitter solution is not allowed. Moreover, contrary to the previous case, 2-fermi and cosmological terms are not enough to render the bulk lagrangian supersymmetric. Closer inspection shows, that terms of the form $\alpha(\epsilon \psi) \partial_{\alpha} \sigma$ do not cancel and the bulk lagrangian must be supplemented with a coupling $\alpha \partial_{\beta} \sigma \mathcal{A}^{\beta}$. In the context of $5 \mathrm{~d}$ supergravity this means that the translations of the pseudoscalar $\sigma$ from the hypermultiplet are gauged, with graviphoton being the gauge field. To recapitulate, after starting with the boundary term (14) we are led to 5 d gauged supergravity similar to that studied in [四].

One could also imagine other powers of V occuring in (14), let us say some function $f(V)$. But then supersymmetrization is possible only if the bulk sigma model quaternionic metric is found. In some simple cases one can appropriately redefine $\operatorname{Re}(\mathrm{S})$ and end up in the same sigma model, however in general one has to search for new sigma models with quaternionic kinetic metric that allow gauging, which is beyond the scope of this paper.

Interestingly enough, we can join both schemes discussed in this paper and demand a boundary term:

$$
\mathcal{L}_{B}=\delta\left(x^{5}\right) \frac{e}{\kappa^{2}}\left(-\Lambda+\frac{\sqrt{2} \alpha}{V}\right) .
$$

As explained we need a similar term on the second brane:

$$
\mathcal{L}_{B^{\prime}}=-\delta\left(x^{5}-\pi \rho\right) \frac{e}{\kappa^{2}}\left(-\Lambda+\frac{\sqrt{2} \alpha}{V}\right)
$$

Repeating the same line of arguments, we arrive at the conlusion, that we need the gauged supergravity in the bulk of the kind considered in [1], but with the potential:

$$
\mathcal{L}_{C}=\frac{e_{5}}{6 \kappa^{2}}\left(-\Lambda+\frac{\sqrt{2} \alpha}{V}\right)^{2}-\frac{e_{5}}{2 \kappa^{2}} \frac{\alpha^{2}}{V^{2}} .
$$

Additional terms are needed to arrive at completely supersymmetric bulk action and they all fit into the general form of gauged supergravity with local translations of $\sigma$.

Since we want to compactify this theory down to $4 \mathrm{~d}$ and demand that the effective theory has $\mathrm{N}=1$ supersymmetry, we must search for the background which preserves exactly four supercharges. The supersymmetry transformation laws of fermions, including modifications 
found in the previous paragraphs are:

$$
\begin{aligned}
\delta \psi_{\alpha}^{A} & =D_{\alpha} \epsilon^{A}-\epsilon\left(x^{5}\right) \frac{1}{12}\left(-\Lambda+\frac{\sqrt{2} \alpha}{V}\right) \gamma_{\alpha}\left(\sigma^{3}\right)_{B}^{A} \epsilon^{B} \\
\delta \lambda^{a} & =-\frac{i}{2 \sqrt{2} V} \partial_{5} V \gamma^{5}\left(\sigma^{3}\right)^{a}{ }_{B} \epsilon^{B}+\alpha \epsilon\left(x^{5}\right) \frac{i}{2 V} \epsilon^{a} .
\end{aligned}
$$

In the above formulas we neglected terms with $4 \mathrm{~d}$ derivatives $\partial_{\mu}$ so as to preserve $4 \mathrm{~d}$ Poincare invariance. We also put $\sigma=\mathcal{A}_{5}=0$ since these fields do not occur in the potential, so this choice is consistent with equations of motion. Finally, we neglected $\partial_{5} \xi$ term since, as we show later in this letter, expectation value of this term generically leads to supersymmetry breaking.

The ansatz for static solutions is: $d s^{2}=a\left(x^{5}\right) d x^{\mu} d x^{\nu} \eta_{\mu \nu}+b\left(x^{5}\right)\left(d x^{5}\right)^{2}, V=V\left(x^{5}\right)$. The relevant supersymmetry transormation laws evaluated for this ansatz are (' denotes $\partial_{5}$ and the world indices are with respect to the Minkowski metric $\eta$ ):

$$
\begin{gathered}
\delta \psi_{\mu}^{A}=\frac{a^{\prime}}{4 \sqrt{a b}} \gamma_{\mu} \gamma_{5} \epsilon^{A}-\epsilon\left(x^{5}\right) \frac{\sqrt{a}}{12}\left(-\Lambda+\frac{\sqrt{2} \alpha}{V}\right) \gamma_{\mu}\left(\sigma^{3}\right)_{B}^{A} \epsilon^{B} \\
\delta \psi_{5}^{A}=\partial_{5} \epsilon^{A}-\epsilon\left(x^{5}\right) \frac{\sqrt{b}}{12}\left(-\Lambda+\frac{\sqrt{2} \alpha}{V}\right) \gamma_{5}\left(\sigma^{3}\right)_{B}^{A} \epsilon^{B} \\
\delta \lambda^{a}=-\frac{i}{2 \sqrt{2 b} V} V^{\prime}\left(\sigma^{3}\right)_{B}^{a} \gamma_{5} \epsilon^{B}+\alpha \epsilon\left(x_{5}\right) \frac{i}{2 V} \epsilon^{a} .
\end{gathered}
$$

We obtain conditions for unbroken supersymmetry by demanding that the above variations of fermionic fields are vanishing for vacuum configurations

$$
\begin{gathered}
\frac{a^{\prime}}{a}=\frac{1}{3}\left(-\Lambda+\frac{\sqrt{2} \alpha}{V}\right) \epsilon\left(x^{5}\right) \sqrt{b} \\
V^{\prime}=\sqrt{2} \alpha \epsilon\left(x^{5}\right) \sqrt{b} \\
\partial_{5} \epsilon^{A}=\frac{\sqrt{b}}{12}\left(-\Lambda+\frac{\sqrt{2} \alpha}{V}\right) \epsilon\left(x^{5}\right) \epsilon^{A} .
\end{gathered}
$$

In addition we need chirality conditions for the supersymmetry generating spinor, which reduce $\mathrm{N}=2$ supersymmetry down to $\mathrm{N}=1$ :

$$
\gamma_{5} \epsilon^{1}=\epsilon^{1} \quad \gamma_{5} \epsilon^{2}=-\epsilon^{2}
$$

It turns out, that if the parameters $a, b, V$ of our ansatz satisfy conditions (25), they automatically satisfy the equations of motion (with delta sources), and give vanishing vacuum energy. We can easily solve the conditions (25). In the coordinate frame where $b=R_{0}^{2}$ the vacuum solution is:

$$
\begin{gathered}
V=V_{0}+\alpha \sqrt{2} R_{0}\left(\left|x^{5}\right|-\frac{\pi \rho}{2}\right) \\
g_{\mu \nu}=\left(1+\alpha \sqrt{2} \frac{R_{0}}{V_{0}}\left(\left|x^{5}\right|-\frac{\pi \rho}{2}\right)\right)^{1 / 3} e^{\frac{-R_{0} \Lambda}{3}\left|x^{5}\right|} \eta_{\mu \nu} \\
g_{55}=R_{0}^{2}
\end{gathered}
$$

where a constant coefficient in the solution for $g_{\mu \nu}$ has been absorbed into a redefinition of the relation between $5 \mathrm{~d}$ and $4 \mathrm{~d}$ Planck scales. With the standard procedure we identify the four-dimensional Planck scale as

$$
M_{4}^{2}=2 M_{5}^{3} R_{0} \int_{0}^{\pi \rho} d x^{5} a\left(x^{5}\right)=2 M_{5}^{3} R_{0} \int_{0}^{\pi \rho} d x^{5}\left(1+\alpha \sqrt{2} R_{0}\left(\left|x^{5}\right|-\frac{\pi \rho}{2}\right)\right)^{1 / 3} \exp \left(-\frac{R_{0}}{3} \Lambda x^{5}\right)
$$


In particular, for $\alpha=0$ one obtains $M_{4}^{2}=\frac{6 M_{5}^{3}}{\Lambda}\left(1-e^{-\frac{\Lambda R_{0} \pi \rho}{3}}\right)$.

For $\Lambda \rightarrow 0$ we are back in the domain wall solution studied in [1], while in the case $\alpha \rightarrow 0$ we get the Randall-Sundrum expotential solution (the connection with the normalization of reference [9] is $\Lambda=6 k$ ). If we assume $\alpha \rho$ to be small (which is the case in the M-theoretical scenario) we are very close to the Randall-Sundrum solution and, in particular, gravity is still localized on the positive tension brane at $x^{5}=0$.

For phenomenological applications we need gauge and charged matter fields transforming in representations of the Standard Model. The problem of coupling confined to a boundary gauge and matter fields to 5 d supergravity can be studied along the lines of the original HoravaWitten procedure [6] and details of this can be found in [15]. We summarize the results. Let us add a gauge multiplet $\left(A_{\mu}^{a}, \chi^{a}\right)$, say, on the first brane ('a' is the group index) and set the kinetic function to $-\frac{V}{4} F_{\mu \nu} F^{\mu \nu}$. It turns out that supersymmetric coupling is possible and no changes in the bulk lagrangian are required. All we need is to add the boundary Lagrangian ( $g^{2}$ is the reference gauge coupling):

$$
\begin{gathered}
\mathcal{L}_{Y M}=\frac{e_{4} \delta\left(x^{5}\right)}{g^{2}}\left(-\frac{V}{4} F_{\mu \nu}^{a} F^{a \mu \nu}-\frac{1}{4} \sigma F_{\mu \nu}^{a} \tilde{F}^{a \mu \nu}-\frac{V}{2} \overline{\chi^{a}} \not D \chi^{a}+\frac{V}{4}\left(\bar{\psi}_{\mu} \gamma^{\nu \rho} \gamma^{\mu} \chi^{a}\right) F_{\nu \rho}^{a}\right. \\
+\frac{3 i}{4 \sqrt{2}} \frac{V}{e_{5}^{5}}\left(\bar{\chi}^{a} \gamma^{5} \gamma^{\mu} \chi^{a}\right) \mathcal{F}_{\mu 5}-\frac{1}{4}\left(\bar{\lambda} \gamma^{\nu \rho} \chi^{a}\right) F_{\nu \rho}^{a}-\frac{i}{8}\left(\bar{\chi}^{a} \gamma^{5} \gamma^{\mu} \chi^{a}\right) \partial_{\mu} \sigma \\
\left.-\frac{\sqrt{V}}{4 e_{5}^{5}}\left(\left(\overline{\chi^{a}}{ }_{L} \chi_{R}^{a}\right) \partial_{5} \bar{\xi}+\left(\overline{\chi^{a}}{ }_{R} \chi_{L}^{a}\right) \partial_{5} \xi\right)+(4 \text { fermi })\right) .
\end{gathered}
$$

In the above the bulk fermions appear in their even (and Majorana in the $4 \mathrm{~d}$ sense) combinations defined as:

$$
\psi_{\mu}=\left(\begin{array}{c}
i \psi_{L \mu}^{2} \\
i \psi_{R \mu}^{1}
\end{array}\right) \quad \psi_{5}=\left(\begin{array}{c}
-i \psi_{L 5}^{1} \\
i \psi_{R 5}^{2}
\end{array}\right) \quad \lambda=\sqrt{2} V\left(\begin{array}{c}
-\lambda_{L}^{1} \\
\lambda_{R}^{2}
\end{array}\right)
$$

We also need to modify the supersymmetry transformation laws of the even bulk fermions:

$$
\begin{gathered}
\delta \psi_{\mu}=\delta\left(x^{5}\right) \frac{\kappa^{2}}{g^{2}} \frac{V}{8}\left(g^{\mu \rho}-\frac{1}{2} \gamma^{\mu \rho}\right) \gamma^{5} \epsilon\left(\overline{\chi^{a}} \gamma^{5} \gamma_{\rho} \chi^{a}\right) \\
\delta \lambda=\delta\left(x^{5}\right) \frac{\kappa^{2}}{g^{2}} \frac{V^{2}}{4}\left(\epsilon\left(\bar{\chi}^{a} \chi^{a}\right)-\gamma^{5} \epsilon\left(\bar{\chi}^{a} \gamma^{5} \chi^{a}\right)\right) .
\end{gathered}
$$

In the above, the supersymmetry parametr $\epsilon$ is defined as an even combination of $5 \mathrm{~d}$ supersymmetry parameters:

$$
\epsilon=\left(\begin{array}{c}
i \epsilon_{L}^{2} \\
i \epsilon_{R}^{1}
\end{array}\right)
$$

Note that no gaugino dependent correction appears in the transformation law of $\psi_{5}$.

In analogy to heterotic models, we have the possibility to break supersymmetry by gaugino condesation on the hidden and/or visible brane. The supersymmetry breaking is transmitted between branes by the expectation value of the hypermultiplet field $\xi$. This mechanism arises because $\xi$, although odd, couples to gauginos on the boundaries through its fifth derivative. The equation of motion for $\xi$ in the presence of the condensates is:

$$
\frac{1}{\kappa^{2}} \partial_{5}\left(\frac{e_{5} g^{55}}{V} \partial_{5} \xi\right)=\partial_{5}\left(-\frac{e_{4} \sqrt{V}}{2 g^{2} e_{5}^{5}}\left(\delta\left(x^{5}\right)\left(\bar{\chi}_{L} \chi_{R}\right)_{1}+\delta\left(x^{5}-\pi \rho\right)\left(\bar{\chi}_{L} \chi_{R}\right)_{2}\right)\right)
$$


We are interested in the solution for $\partial_{5} \xi$ because this expression (and not $\xi$ alone) appears in the relevant formulae. For $\alpha=0$ the solution is :

$$
\partial_{5} \xi=-\frac{\kappa^{2}}{2 g^{2}} V_{0}^{3 / 2}\left(\delta\left(x^{5}\right) \chi_{1}^{2}+\delta\left(x^{5}-\pi \rho\right) \chi_{2}^{2}\right)+C \exp \left(\frac{2 R_{0}}{3} \Lambda|y|\right) .
$$

The non-trivial background effects are due to the expotential factors. The constant $\mathrm{C}$ can be determined from the boundary conditions (in other words, from matching delta singularities in the equation of motion):

$$
C=\frac{R_{0} \Lambda}{e^{\frac{2 R_{0}}{3} \Lambda \pi \rho}-1} \frac{\kappa^{2}}{6 g^{2}} V_{0}^{3 / 2}\left(\chi_{1}^{2}+\chi_{2}^{2}\right) .
$$

For $\Lambda=0$ it is customary [1] to go to a different coordinate frame where $g_{55}=R_{0}^{2} H^{4}, g_{\mu \nu}=$ $\frac{1}{R_{0}} H \bar{g}_{\mu \nu}, V=V_{0} H^{3}$ and $H=1+\frac{\alpha \sqrt{2} \pi \rho R_{0}}{3 V_{0}}\left(\left|x^{5}\right|-\frac{\pi \rho}{2}\right)$. In this frame we obtain the solution:

$$
\begin{gathered}
\partial^{5} \xi H^{-3}=\frac{\kappa^{2}}{2 g^{2}} V_{0}^{3 / 2} H^{3 / 2}\left(-\delta\left(x^{5}\right) \chi_{1}^{2}-\delta\left(x^{5}-\pi \rho\right) \chi_{2}^{2}\right)+C \\
C=\frac{\kappa^{2}}{3 g^{2}} \alpha \sqrt{2} \pi \rho V_{0}^{3 / 2} \frac{-\chi_{1}^{2} H^{9 / 2}(0)-\chi_{2}^{2} H^{9 / 2}(\pi \rho)}{H^{4}(0)-H^{4}(\pi \rho)} .
\end{gathered}
$$

It is worth noting, that in the $5 \mathrm{~d}$ theory gaugino condensates break supersymmetry (but, if we assume superpotentials on the branes we can cancel their contribution). In the presence of the condensates we have no way to satisfy simultanously $\delta \psi_{\mu}^{A}=0$ and neither of the remaining conditions for unbroken supersymmetry. Indeed, $\partial_{5} \xi$ and condensates do not alter the transformation law of $\psi_{\mu}$, so in particular, the conditions resulting from $\delta \psi_{\mu}^{A}=0$ include chirality conditions (26). But then, the condensates in $\delta \lambda^{a}$ and $\delta \psi_{5}^{A}$ multiply the supersymmetry parameter $\epsilon$, which is of the chirality opposite to other $\epsilon$ 's occuring in these transformation laws. Thus, conditions $\delta \psi_{5}^{A}=0$ and $\delta \lambda^{a}=0$ cannot be satisfied.

When we compactify our model to $4 \mathrm{~d}$ on the background (27), the independent of $x^{5}$ integration constants $R_{0}, V_{0}$ together with zero modes of $\sigma$ and $\mathcal{A}_{5}$, become the ( $x^{\mu}$ dependent) moduli of the effective $4 \mathrm{~d}$ theory. The fluctuations around Minkowski metric in the solution (27) are described by $\bar{g}_{\mu \nu}$ To go to the $4 \mathrm{~d}$ Einstein frame one needs to perform explicit integration over $x^{5}$ and a suitable moduli dependent Weyl rotation. We rescale the metric $\bar{g}_{\mu \nu} \rightarrow a_{0} \bar{g}_{\mu \nu}$ with $a_{0}$ chosen (up to a numerical, independent of moduli, factor which can be absorbed into the definition of the $4 \mathrm{~d}$ gravitational constant) as $a_{0}^{-1}=\int_{0}^{2 \pi \rho} d x^{5} a\left(x^{5}\right)$. The Killing spinors generating an unbroken $N=1$ supersymmetry are:

$$
\begin{aligned}
& \epsilon_{R}^{1}=e^{-\frac{\Lambda R_{0}\left|x^{5}\right|}{12}}\left(1+\alpha \sqrt{2} \frac{R_{0}}{V_{0}}\left(\left|x^{5}\right|-\frac{\pi \rho}{2}\right)\right)^{1 / 12} a_{0}^{-1 / 4} \eta_{R} \\
& \epsilon_{L}^{2}=e^{-\frac{\Lambda R_{0}\left|x^{5}\right|}{12}}\left(1+\alpha \sqrt{2} \frac{R_{0}}{V_{0}}\left(\left|x^{5}\right|-\frac{\pi \rho}{2}\right)\right)^{1 / 12} a_{0}^{-1 / 4} \eta_{L} .
\end{aligned}
$$

Since $\epsilon_{L}^{2}=-i \sigma^{2} \epsilon_{R}^{1 *}(5 \mathrm{~d}$ Majorana condition) spinor $\eta$ is Majorana in the $4 \mathrm{~d}$ sense. The factor $a_{0}$ in (38) yields canonical form of the reduced $4 \mathrm{~d}$ supersymmetry transformation law of the gravity multiplet, $\eta$ depends only on $x^{\mu}$ and has an interpretation of a parameter of supersymmetry transformations in the $4 \mathrm{~d}$ theory. 
When $\Lambda \neq 0$ it is pretty difficult to compactify the supersymmetric model which we have defined to four dimensions and even more to bring it into the standard $4 \mathrm{~d}$ supergravity form. Hence we postpone the discussion of this case for a while and discuss first in detail the simpler case with $\Lambda=0$ and $\alpha \neq 0$. We can now proceed with the derivation of the $4 \mathrm{~d}$ effective theory. In that case the Kähler potental is [1]:

$$
K=-\ln (S+\bar{S})-3 \ln (T+\bar{T}) .
$$

The moduli $S, T$ and their superpartners are defined as:

$$
\begin{array}{cc}
S=V_{0}+i \sigma_{0} & \Lambda^{S}=<\left(\frac{H}{R_{0}}\right)^{1 / 4}\left(\lambda-\alpha \sqrt{2}\left(\left|x^{5}\right|-\pi \rho\right) \psi_{5}\right)> \\
T=R_{0}+i \sqrt{2} \mathcal{A}_{5} & \Lambda^{T}=<\left(\frac{H}{R_{0}}\right)^{1 / 4} \psi_{5}>
\end{array}
$$

where $\langle(\ldots)\rangle=\frac{1}{2 \pi \rho} \int d x^{5}(\ldots)$. The gauge sectors originating from two different branes are described by the gauge functions, which includes corrections linear in $\alpha$ :

$$
\begin{aligned}
& f_{1}=S-\frac{\sqrt{2}}{2} \alpha \pi \rho T \\
& f_{2}=S+\frac{\sqrt{2}}{2} \alpha \pi \rho T .
\end{aligned}
$$

The physical gauginos can be expressed as $\left(\chi_{1}\right)_{p}=\left(\chi_{1}\right)\left(\frac{H(0)}{R_{0}}\right)^{3 / 4},\left(\chi_{2}\right)_{p}=\left(\chi_{2}\right)\left(\frac{H(\pi \rho)}{R_{0}}\right)^{3 / 4}$.

The above corrections were extracted from the kinetic terms of the 5 d lagrangian compactified to $4 \mathrm{~d}$. Although the functions $\mathrm{K}$ and $\mathrm{f}$ are sufficient to reconstruct the rest of the supergravity lagrangian, an interesting consistency check would be to obtain explicitly the complete 4d lagrangian by integrating out the fifth dimension. This is fairly difficult as, e.g., the 4 -fermi terms have higher order in $\alpha$ contributions. Another approach is to reduce $5 \mathrm{~d}$ supersymmetry transformation laws to $4 \mathrm{~d}$, and check if they are consistent with the results (39,41). This has the advantage that corrections can be seen at lower order in the expansion in $\alpha$ and $\kappa^{2}$. As an example we present how to determine the gauge kinetic functions from the transformation laws of moduli superpartners. We use the definition of $S$ and $T$ superpartners (40) and substitute $\partial_{5} \xi$ with the solution of its equation of motion in the relevant part of $5 \mathrm{~d}$ supersymmetry transformation law of $\lambda$ and $\psi_{5}$. After integrating over fifth dimension the result up to $\alpha^{2}$ corrections is:

$$
\begin{array}{r}
\delta \Lambda_{L}^{S}=\frac{\kappa_{4}^{2}}{2 g^{2}} V_{0}^{2}\left(\chi_{1}^{2}+\chi_{2}^{2}\right) \eta_{L} \\
\delta \Lambda_{L}^{T}=-\frac{\kappa_{4}^{2}}{12 g^{2}} R_{0}^{2} \alpha \sqrt{2} \pi \rho\left(\chi_{1}^{2}-\chi_{2}^{2}\right) \eta_{L} .
\end{array}
$$

Noting that in $4 \mathrm{~d}$ supergravity, scalar gaugino condensates in the transformation law of the fermions $\Lambda^{S}, \Lambda^{T}$ are multiplied by $\frac{1}{8} f,_{S}\left(K^{-1}\right)_{S}^{S}$ and $\frac{1}{8} f,_{T}\left(K^{-1}\right)_{T}^{T}$, respectively, the result indeed agrees with (41). A noteworthy detail in this derivation is that in $5 \mathrm{~d} \partial_{5} \xi$ appears as a full square: $\partial_{5} \hat{\xi}=\partial_{5} \xi+\frac{\kappa^{2}}{g^{2}} \delta\left(x^{5}\right) \frac{V^{3 / 2}}{2}\left(\left(\overline{\chi_{R}} \chi_{L}\right)_{1}+\left(\overline{\chi_{R}} \chi_{L}\right)_{2}\right)$ in $\delta \lambda$ but not in $\delta \psi_{5}$. Thus, when we calculate $\delta \Lambda^{T}$ the linear part of the solution for $\partial_{5} \xi$ cancels to zeroth order in $\alpha$ with delta functions 
occuring in this solution, leading to the correct form of $f_{, T}$. Note also, that the admixture of $\psi_{5}$ in the definition of $\Lambda^{S}$ is crucial to obtain the correct form of $f_{, S}$. These conclusions confirm fully the results of [3, 4,8$]$.

From the transformation laws (42) it can be read off that presence of gaugino condensates breaks supersymmetry also in the $4 \mathrm{~d}$ effective theory. Although one can adjust $\chi_{1}^{2}=-\chi_{2}^{2}$ so that the condesates cancel in the regular part of the solution (36) for $\partial_{5} \xi$ and in consequence in $\delta \Lambda^{S}$, but then the non-zero condensate contribution appears in $\delta \Lambda^{T}$ due to the above mentioned lacking of the 'full square' structure of $\delta \psi_{5}$. However, if we allow for boundary scalar fields, by appropriate adjusting of their superpotentials we have the possibility to cancell the contribution of the condensates.

To be precise, we note that the above cosiderations for the case $\Lambda=0$ are valid only to linear order in $\alpha$. In $\alpha^{n}$ order, with $n>1$, further corrections appear, but they are difficult to calculate. One needs to solve the equations of motion for KK modes of the bulk fields (to linear order in $\alpha$ it suffices to know the expectation value of the bulk fields on the branes).

While neglecting the higher order corrections in $\alpha$ can be justified by the expected smallness the expansion parameter $\alpha \pi \rho$, this is not the case for $\Lambda \pi \rho$, since $\Lambda$ is expected to be set by the string scale. Thus, finding the effective theory with a non-zero $\Lambda$, even for the case $\alpha=0$, requires more elaborate tools. However, although we do not know the complete effective Lagrangian, we can still try to extract some information about the low energy $4 \mathrm{~d}$ theory by computing physically important $4 \mathrm{~d}$ operators, and using experience gained in the study of the simpler model. Let us put $\alpha=0$ in what follows. We know already that the parameter controlling the breakdown of low energy supersymmetry is the regular part of $\partial_{5} \xi$ given in formula (36). This equals in the present case $\partial_{5} \xi\left(x^{5}\right)=\frac{1}{e^{\frac{2}{3} R_{0} \Lambda \pi \rho}-1} \frac{\kappa^{2} R_{0} \Lambda}{6 g^{2}} V_{0}^{3 / 2}\left(\chi_{1}^{2}+\chi_{2}^{2}\right) e^{\frac{2}{3} R_{0} \Lambda\left|x^{5}\right|}$.

We note that in the presence of gaugino codensates the vacuum expectation value of $\partial_{5} \xi$ is nonzero, and is modulated by an expotential, $x^{5}$-dependent factor. The above expression can be inserted into the bulk kinetic term of $\partial_{5} \xi$ (the singular part of the solution drops out due to the 'full square' structure), to read off physical gaugino masses on each wall. These are the important parameters as they can tell us directly the physical magnitudes of induced global supersymmetry breaking terms in the boundary gauge sectors. In the limiting case $\Lambda \pi \rho \ll 1$, to the lowest order (and keeping $\alpha=0$ ), we recover this way soft gaugino masses which we have obtained in the compactification of the pure M-theoretical model. To perform the task in a general case, we need to expand metric around the vacuum solution. At the same time, to obtain kanonical normalization of kinetic terms of fields living on the branes we need to rescale them by expotential factors. This also gives the standard form of the supersymmetry transformation law of the gauge field $A_{\mu}$ reduced in our background, $\delta A_{\mu}=\bar{\eta} \gamma_{\mu} \chi$. The needed rescaling is $\chi_{i}=a_{i}^{-3 / 4}\left(\chi_{i}\right)_{p}$, i.e. $\left(\chi_{1}\right)_{5 d}=a_{0}^{-3 / 4}\left(\chi_{1}\right)_{p}$ and $\left(\chi_{2}\right)_{5 d}=a_{0}^{-3 / 4} e^{\frac{1}{4} R_{0} \Lambda \pi \rho}\left(\chi_{2}\right)_{p}$. If we interpret quartic gaugino terms as leading to gaugino masses after condensation the result for the masses is (in the limit $\Lambda \pi \rho \gg 1$ ) ?.

$$
\begin{aligned}
& M_{1}=\frac{V_{0} \kappa_{4}^{2}}{2 g^{4}} e^{-\frac{2}{3} R_{0} \Lambda \pi \rho}\left(<\chi_{1 p}^{2}>+<\chi_{2 p}^{2}>e^{\frac{1}{2} R_{0} \Lambda \pi \rho}\right) \\
& M_{2}=\frac{V_{0} \kappa_{4}^{2}}{2 g^{4}} e^{-\frac{1}{6} R_{0} \Lambda \pi \rho}\left(<\chi_{1 p}^{2}>+<\chi_{2 p}^{2}>e^{\frac{1}{2} R_{0} \Lambda \pi \rho}\right) .
\end{aligned}
$$

\footnotetext{
${ }^{2}$ It is useful to express the $5 \mathrm{~d}$ gravitational coupling $\kappa^{2}$ through the $4 \mathrm{~d}$ coupling $\kappa_{4}^{2}$. In the two cases of interest the relation is a) $\kappa_{4}^{2}=\kappa^{2} /(2 \pi \rho)$ when $\Lambda \pi \rho \ll 1$ and b) $\kappa_{4}^{2}=\kappa^{2} \Lambda / 6$ when $\Lambda \pi \rho \gg 1$.
} 
For definitness of the discussion, let us consider one after another two possible visible-hidden sector configurations. First we put the visible sector on the negative tension brane at $x^{5}=\pi \rho$ and switch on the hidden condensate on the positive tension brane. First of all, we see that $M_{2} \approx e^{-\frac{1}{6} R_{0} \Lambda \pi \rho}<\chi_{1 p}^{2}>$ becomes exponentially suppressed as a function of the distance between walls, and it scales exactly as expected on the basis of the argument given in [10]. If we now revert the roles of the branes, and assume that the positive tension brane contains observable fields, the situation is similar, i.e. $M_{1} \approx e^{-\frac{1}{6} R_{0} \Lambda \pi \rho}<\chi_{2 p}^{2}>$ vanishes with growing distance between the walls. The thing to be noted in this case, which can be called standard hidden sector scenario, is the presence of the additional exponential factor in front of the usual dynamically generated mass scale $a^{1 / 2}(\pi \rho) \Lambda_{h i d}^{3} / M_{P l}^{2}$, which is the (not necessarily welcome) source of additional hierarchy. In conclusion we stress that in the limit of large warp factors the transmission of supersymmetry breaking from the hidden brane is expotentially suppresed. Thus, in this scenario walls indeed decouple with the growing distance between them.

A different role of the warp factor can be seen when one considers a condensate forming on the same wall where the observable sector lives. If this happens on the negative tension wall the induced gaugino mass seems to be exponentially enhanced, like $e^{\frac{1}{3} R_{0} \Lambda \pi \rho}$. If on the other hand the visible brane is the positive tension one, the supersymmetry breaking mass is suppressed by the factor $e^{-\frac{2}{3} R_{0} \Lambda \pi \rho}$. One obvious comment on this is that the walls are not equivalent, in the sense of being interchangable, as was already the case in the nonsupersymmetric RandallSundrum scenario. Second, let us note that expecting large, say well above $1 \mathrm{TeV}^{3}$, condensates on the negative tension brane where all physical scales are scaled down to say $1 \mathrm{TeV}$ may be inconsistent in the present framework.

At this point we should consider matter fields on the boundaries, interacting by means of a trilinear superpotential $W$. New terms in the Lagrangian which should be taken into account are

$$
S_{\text {scalar }}=\int d^{5} x \frac{e_{4}}{g^{2}} \delta\left(x^{5}\right)\left(-D_{\mu} \Phi D^{\mu} \bar{\Phi}-\frac{2}{V} \frac{\partial W}{\partial \Phi} \frac{\partial \bar{W}}{\partial \bar{\Phi}}-\frac{4 \kappa^{2}}{V} W \bar{W}+\frac{2}{V e_{5}^{5}} W \partial_{5} \xi+\text { h.c. }\right)
$$

(and similarly for the second wall). The results of the coupling between $W$ and $\partial_{5} \xi$ are twofold. First, in the previous formulae for the vacuum solution for $\partial_{5} \xi$ one should substitute

$$
\chi_{i} \bar{\chi}_{i} \rightarrow \chi_{i} \bar{\chi}_{i}-\frac{4 W_{i}}{V^{3 / 2}}
$$

This means that expectation value of $W$ contributes to the supersymmetry breaking, and can in principle cancel the contribution of condensates. Let us note, that the canonical normalization of kinetic terms of scalars $\Phi_{i}$ living on the $i$-th wall leads to rescaling $\Phi_{1} \rightarrow \Phi_{1 p} a_{0}^{-1 / 2}$, $\Phi_{2} \rightarrow \Phi_{2 p} a_{0}^{-1 / 2} e^{\frac{1}{6} R_{0} \Lambda \pi \rho}$. This implies that superpotential from the $i$-th wall scales like a condensate from the same wall, hence the earlier discussion of decoupling applies here without modifications.

The second result of new couplings is the appearance of softly breaking global supersymmetry trilinear scalar terms when condensates are switched on. These terms, say on the second brane, are proportional to

$$
a_{2}^{1 / 2} \frac{1}{V e_{5}^{5}} W_{2 p}<\chi_{p}^{1}-\frac{4 W_{1 p}}{V^{3 / 2}}>
$$


and one can easily work out their scaling properties. These terms are the physical soft terms assuming that the effective $4 \mathrm{~d}$ vacuum energy, after switching on vevs for boundary scalars, vanishes.

After presenting this preliminary and somewhat speculative interpretation of the supersymmetry breaking pattern, we want to stress that definite conclusions can be made only when one constructs the complete effective $4 \mathrm{~d}$ theory.

To put this discussion into a wider framework, let us remind ourselves that what we have done here so far is the traditional compactification of the fifth dimension, where one tries to combine both, in principle different, gauge sectors at the ends of the five-dimensional world into a single effective theory. However, a different approach is possible, see [16]. One can imagine that the model living on the negative tension brane located at $x^{5}=\pi \rho$ is a holographic image of the same model living on the Planck brane located at $x^{5}=0$. Flow of the second brane along the $x^{5}$ axis accompanied by rescaling of all the mass scales on that brane by a factor $a^{1 / 2}\left(x^{5}\right)$ might be considered to be equivalent to renormalization group flow along momentum scale towards the IR limit. In this context we want to notice, that in the $N=1$ theory which we study here the mass scales scale exactly in the way required by holographic principle, but the gauge coupling does not scale with the changing warp factor. This is easy to see, since the warp factor cancels out from the expression $e_{4} g^{\mu \nu} g^{\beta \alpha} F_{\mu \beta} F_{\nu \alpha}$. The intriguing observation is that if one would try to improve for that, and scale also the gauge coupling according to one-loop scaling anomaly, see [17], $\frac{1}{g^{2}\left(x^{5}\right)}=\frac{1}{g^{2}\left(M_{5}\right)}+b_{0} \log \left(\frac{M_{5}}{m\left(x^{5}\right)}\right)^{2}=\frac{1}{g^{2}\left(M_{5}\right)}-b_{0} \log a\left(x^{5}\right)$, then assuming $\frac{1}{g^{2}\left(x^{5}\right)}=\frac{1}{g_{G U T}^{2}\left(x^{5}\right)}$ this flow would compensate the relative enhancement factor between gaugino condensate from the second and first wall, $\Lambda_{\text {cond }}(\pi \rho)=M_{5} e^{-\frac{1}{2 b_{0}}\left(\frac{1}{g^{2}}-b_{0} \log (a(\pi \rho))\right)} \approx a^{1 / 2}(\pi \rho) \Lambda_{\text {cond }}(0)$.

At the end we would like to comment on two aspects of the models we discuss in this paper. Firstly, it is interesting to note that the additional terms which we have put on the boundary, $\delta L=e f(V)$, are not parts of a globally supersymmetric sigma model living on a brane. When one integrates over the fifth dimension these terms cancel against the bulk potential and drop out completely from the effective four dimensional model. Hence, the presence of the additional dimension offers the possibility of supersymmetrizing certain boundary terms along the direction transverse to the branes, with partner terms living in the bulk. Secondly, the vanishing vacuum energy in the pure bulk moduli sector which we observe does not solve automatically the cosmological constant problem. When we allow matter chiral superfields on the branes to follow their local dynamics given by nontrivial superpotential and gauge interactions, the new vacuum they approch is not guaranteed to give automatically a vanishing contribution to the $4 \mathrm{~d}$ vacuum energy, and in general next instance of tuning is necessary.

To summarize, we have presented a class of five dimensional supergravities with gauge sectors living on $4 \mathrm{~d}$ boundaries, which admit exponential warp factors analogous to that of the Randall-Sundrum model. The required fine-tuning between bulk and boundary cosmological potetnials was explained by supersymmetry. These models can be considered to be deformations of the M-theoretical model constructed in [1]. We have discussed hidden sector supersymmetry breaking and its transmission between branes in the present models. The setup and results are likely to be relevant for the discussion of the holographic projection of $N=1$ supersymmetric gauge models. 
This work has been supported by TMR programs ERBFMRX-CT96-0045 and CT96-0090. Z.L. and S.P. are supported by the Polish Committee for Scientific Research grant 2 P03B 05216(99-2000).

\section{References}

[1] A. Lukas, B. A. Ovrut, K. S. Stelle and D. Waldram, Phys. Rev. D59 (1999) 086001.

[2] A. Lukas, B. A. Ovrut, K. S. Stelle and D. Waldram, Nucl. Phys. B552 (1999) 246.

[3] J. Ellis, Z. Lalak, S. Pokorski, W. Pokorski, Nucl.Phys. B540 (1999) 149.

[4] J. Ellis, Z. Lalak, W. Pokorski, Nucl.Phys. B559 (1999) 71.

[5] E. Witten, Nucl.Phys. B471 (1996) 135.

[6] P. Horava and E. Witten, Nucl.Phys. B460 (1996) 506 and Nucl.Phys. B475 (1996) 94.

[7] T. Banks, M. Dine, Nucl. Phys. B479 (1996) 173; H.P. Nilles, S. Stieberger, Nucl. Phys. B499 (1997) 3; I. Antoniadis, M. Quiros, Nucl. Phys. B505 (1997) 109; T. Li, J. L. Lopez, D.V. Nanopoulos, Phys. Rev. D56 (1997) 2602; E. Dudas, Ch. Grojean, Nucl. Phys. B507 (1997) 553; H.P. Nilles, M. Olechowski, M. Yamaguchi, Phys. Lett. B415 (1997) 24; Z. Lalak, S. Thomas, Nucl. Phys. B515 (1998) 55; A. Lukas, B. Ovrut, D. Waldram, Nucl. Phys. B532 (1998) 43; K. Choi, H. B. Kim, C. Munoz, Phys. Rev. D57 (1998) 7521.

[8] J. Ellis, Z. Lalak, S. Pokorski, S. Thomas, Nucl.Phys. B563 (1999) 107.

[9] L. Randall and R. Sundrum, Phys. Rev. Lett. 83 (1999) 4690.

[10] L. Randall and R. Sundrum, Phys. Rev. Lett. 83 (1999) 3370.

[11] R. Kalosh, A. Linde, JHEP 02 (2000) 0005.

[12] K. Behrndt, M. Cvetić, Phys. Rev. D61 (2000) 101901.

[13] R. Altendorfer, J. Bagger, D. Nemeschansky Supersymmetric Randall-Sundrum scenario, hep-th/0003117.

[14] T. Gherghetta, A. Pomarol, Bulk fields and supersymmetry in a slice of AdS, hepth/0003129.

[15] A. Falkowski, to be published.

[16] H. Verlinde, Holography and Compactification, hep-th/9906182; Supersymmetry at Large Distance Scales, hep-th/0004003.

[17] K. Dienes, E. Dudas, T. Gherghetta, Anomaly Induced Gauge Unification and Brane/Bulk Couplings in Gravity-Localized Theories, hep-th/9908530. 